\title{
Waterlike anomalies in a two-dimensional core-softened potential
}

\author{
José Rafael Bordin ${ }^{1, *}$ and Marcia C. Barbosa ${ }^{2, \dagger}$ \\ ${ }^{1}$ Campus Caçapava do Sul, Universidade Federal do Pampa, Avenida Pedro Anunciação, 111, \\ CEP 96570-000 Caçapava do Sul, Rio Grande do Sul, Brazil \\ ${ }^{2}$ Instituto de Física, Universidade Federal do Rio Grande do Sul, Caixa Postal 15051, CEP 91501-970 Porto Alegre, Rio Grande do Sul, Brazil
}

(Received 27 December 2017; published 9 February 2018)

\begin{abstract}
We investigate the structural, thermodynamic, and dynamic behavior of a two-dimensional (2D) core-corona system using Langevin dynamics simulations. The particles are modeled by employing a core-softened potential which exhibits waterlike anomalies in three dimensions. In previous studies in a quasi-2D system a new region in the pressure versus temperature phase diagram of structural anomalies was observed. Here we show that for the two-dimensional case two regions in the pressure versus temperature phase diagram with structural, density, and diffusion anomalies are observed. Our findings indicate that, while the anomalous region at lower densities is due the competition between the two length scales in the potential at higher densities, the anomalous region is related to the reentrance of the melting line.
\end{abstract}

DOI: 10.1103/PhysRevE.97.022604

\section{INTRODUCTION}

Anomalous materials show characteristics that differ from those observed in most substances. For instance, it is expected that liquids contract upon cooling at constant pressure and diffuse slower upon compression. However, anomalous fluids expand as the temperature is decreased and move faster as the pressure grows. The most well known anomalous system is water, with more than 70 known anomalies [1], but there are another anomalous fluids. The maximum in the diffusion coefficient at constant temperature was observed not only for water [2] but also for silicon [3] and silica [4]. The maximum in the density well known in water [5] is also seen in silicon [4], silica [6], Te [7], Bi [8], Si [9], $\mathrm{Ge}_{15} \mathrm{Te}_{85}$ [10], liquid metals [11], graphite [12] and $\mathrm{BeF}_{2}$ [13].

Since the seminal work by Jagla [14-16], core-softened potentials have been widely used in the literature to study the behavior of anomalous fluids [17-24]. The waterlike anomalies were observed in potentials with two characteristic length scales [25,26], as well as softened repulsive potentials [27-29]. In both cases the competition between distinct fluid conformations can be directly related to the anomalies [29,30]. In addition, these models have been employed to understand the transition between a high density liquid (HDL) and a low density liquid (LDL): the liquid-liquid phase transition (LLPT) [31-35]. Theoretically, the existence of these two liquid phases was evidenced in the ST4 model of water by Poole and co-authors [36] and in models for phosphorus [37], silica [38,39], silicon [40], carbon [41], hydrogen [42], and colloidal systems [43].

Core-softened potentials have also been applied to study colloidal systems. Experimental works have shown that the effective interaction between colloids can be modeled by

\footnotetext{
*josebordin@unipampa.edu.br

${ }^{\dagger}$ marcia.barbosa@ufrgs.br
}

core-softened potentials $[44,45]$. The origin of the two length scales in these macromolecules goes as follows. The colloids are usually made of molecular subunits which form a central packed agglomeration and a less dense and more entropic peripheral area. This core-corona structure can be described by a hard core and a soft corona. Then it becomes natural to model the system by a two-length-scales potential, which leads to the self-assembled patterns observed in these colloidal systems [46-56].

Interesting aspects of two-dimensional (2D) colloidal suspensions include the self-assembly and the distinct patterns observed in these systems [50-55,57,58]. Also, the anomalous melting scenario, the existence of a hexatic phase [59-61], and dynamics of the fluid phase $[47,56,62-64]$ of these twodimensional macromolecule systems have attracted a lot of attention. Given the wide spectrum of the properties observed in the 2D core-softened systems, in this paper we focus only on the fluid phase. We propose to clarify how the waterlike anomalies in these 2D core-corona systems behave for different pressures and temperatures and particularly what is the effect of exposing these macromolecules to a solvent.

In order to address this question, we study a system in which the interparticle colloid-colloid interaction has a repulsive core with a smooth shoulder. The core-softened molecular systems in the $3 \mathrm{D}$ bulk $[20,65]$ and in the confined quasi-2D structure [66-68] show waterlike anomalies. In the particular case of the quasi-2D structure, in addition to the waterlike anomalous region in the pressure versus temperature phase diagram, a new region of structural anomaly was observed [69]. Here we show that for the $2 \mathrm{D}$ systems the molecular system presents a second region of anomalies in the pressure versus temperature phase diagram. A mechanism for the appearance of this second anomalous region is proposed.

Our paper is organized as follows. In Sec. II the model and the details about the simulation method are presented. In Sec. III results are discussed. The conclusions are given in Sec. IV. 


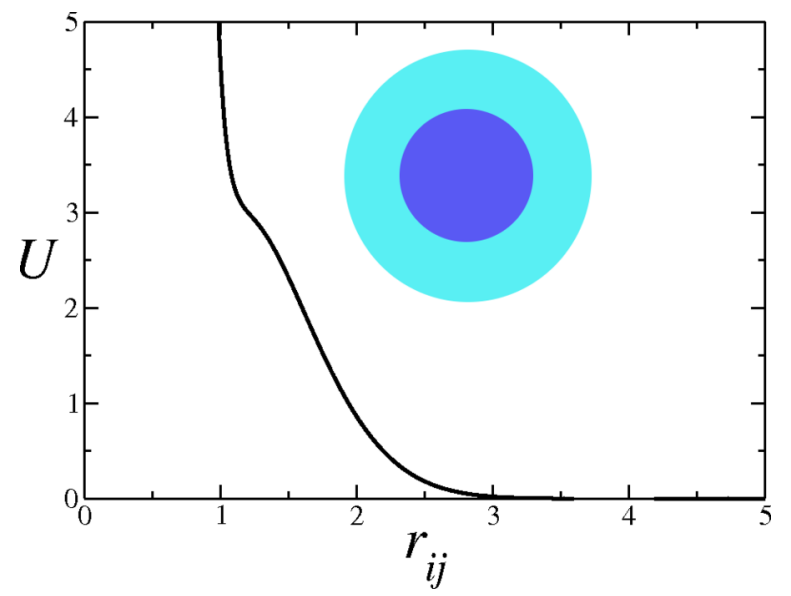

FIG. 1. Core-softened interaction potential $U$ between two corecorona particles. Inset: schematic depiction of the particles, with the core (first length scale at $r_{i j} \equiv r_{1} \approx 1.2 \sigma$ ) and the soft corona (second length scale at $r_{i j} \equiv r_{1} \approx 2.0 \sigma$ ).

\section{THE MODEL AND THE SIMULATION DETAILS}

For simplicity, in this paper all the physical quantities are computed and displayed in the standard Lennard-Jones (LJ) reduced units [70]. The system consists of $N=2000$ disks with diameter $\sigma$ and mass $m$ with a potential interaction composed of a short-range attractive Lennard-Jones potential and a Gaussian term centered in $r_{0}$, with depth $u_{0}$ and width $c_{0}$ :

$$
\begin{aligned}
U\left(r_{i j}\right)= & 4 \epsilon\left[\left(\frac{\sigma}{r_{i j}}\right)^{12}-\left(\frac{\sigma}{r_{i j}}\right)^{6}\right] \\
& +u_{0} \exp \left[-\frac{1}{c_{0}^{2}}\left(\frac{r_{i j}-r_{0}}{\sigma}\right)^{2}\right],
\end{aligned}
$$

where $r_{i j}=\left|\vec{r}_{i}-\vec{r}_{j}\right|$ is the distance between two disks $i$ and $j$. This potential can be parametrized to have a ramplike shape, and was extensively applied to study systems with waterlike anomalies $[20,65]$. The parameters used in this work are $u_{0}=$ $5.0, c=1.0$, and $r_{0} / \sigma=0.7$. The interaction potential, shown in Fig. 1 , has two length scales. The first scale is at $r_{i j} \equiv r_{1} \approx$ $1.2 \sigma$, where the force has a local minimum, and the other scale os at $r_{i j} \equiv r_{2} \approx 2 \sigma$, where the fraction of imaginary modes of the instantaneous normal modes spectra has a local minimum [71]. The cutoff radius for the interaction is $r_{c}=3.5$. The two length scales in the potential allow for using this potential to represent the interaction between hard core-soft shell colloids [51,57].

In this work we use the Langevin thermostat [70] to mimic the solvent effects. Hydrodynamics interactions were neglected. Since the system is in equilibrium we do not expect that this will change the long-time behavior. The temperature was simulated in the interval between $T=0.01$ and $T=0.40$. The number density is defined as $\rho=N / A$, where $A=L^{2}$ is the area and $L$ the size of the simulation box in the $x$ and $y$ directions. $\rho$ was varied from $\rho=0.05$ up to $\rho=0.60$, and the size of the simulation box was obtained via $L=(N / \rho)^{1 / 2}$. For clarity, in the $p \times T$ phase diagram the higher isochore shown is $\rho=0.525$ since no anomalous behavior was observed above this density.

The time step used in the simulations was $\delta t=0.001$, and periodic boundary conditions were applied in the two directions. We performed $3 \times 10^{7}$ steps to equilibrate the system. These steps were then followed by $5 \times 10^{7}$ steps for the results production stage. To ensure that the system was equilibrated, the pressure, kinetic, and potential energy were analyzed as functions of time. Snapshots of the system were also used to verify the equilibration. Also, two distinct initial configurations were used for each point: a random fluidlike configuration and a solidlike configuration in a square lattice. The results obtained were independent of the initial configuration.

To study the dynamic anomaly, the relation between the mean square displacement (MSD) with time was computed, namely

$$
\left\langle\left[\vec{r}(t)-\vec{r}\left(t_{0}\right)\right]^{2}\right\rangle=\left\langle\Delta \vec{r}(t)^{2}\right\rangle,
$$

where $\quad \vec{r}\left(t_{0}\right)=\left[x\left(t_{0}\right)^{2}+y\left(t_{0}\right)^{2}\right]^{1 / 2} \quad$ and $\quad \vec{r}(t)=\left[x(t)^{2}+\right.$ $\left.y(t)^{2}\right]^{1 / 2}$ denote the coordinate of the particle at a time $t_{0}$ and at a later time $t$, respectively. The MSD is related to the diffusion coefficient $D$ by

$$
D=\lim _{t \rightarrow \infty} \frac{\left\langle\Delta \vec{r}(t)^{2}\right\rangle}{4 t} .
$$

The structure of the fluid was analyzed using the radial distribution function (RDF) $g\left(r_{i j}\right)$, and the pressure was evaluated using the virial expression. Directly related to the $g\left(r_{i j}\right)$, the structural anomaly was characterized using the translational order parameter $\tau$, defined as [72]

$$
\tau \equiv \int_{0}^{\xi_{c}}|g(\xi)-1| d \xi
$$

where $\xi=r \rho^{1 / 2}$ is the interparticle distance $r$ divided by the mean separation between pairs of particles $\rho^{1 / 2} . \xi_{c}$ is a cutoff distance, defined as $\xi_{c}=L \rho^{1 / 2} / 2$. For an ideal gas (completely uncorrelated fluid), $g(\xi)=1$ and $\tau$ vanish. For the crystal or the ordered fluids, a translational long order $[g(\xi) \neq 1]$ persists over long distances, increasing the value of $\tau$. Therefore, for normal liquids $\tau$ increases with the increase of the density.

In order to check if the system exhibits the density anomaly, the temperature of maximum density (TMD) was computed for different isochores as follows. Using thermodynamical relations, the TMD was characterized by the minimum in the pressure versus temperature diagram along isochores,

$$
\left(\frac{\partial p}{\partial T}\right)_{\rho}=0 .
$$

The separation between the fluid and the amorphous solid phases was defined by the analysis of the total energy, RDF, MSD, and system snapshots. When the particles showed a well defined structure and had a very low or zero mobility, the phase was defined as solid. When the system exhibited nonzero mobility, it was considered to be in the fluid phase. These phase boundaries were confirmed by the evaluation of the heat capacity [70]. The results were supported by larger simulations, using using $N=5000$ disks and $5 \times 10^{9}$ steps. 
(a)

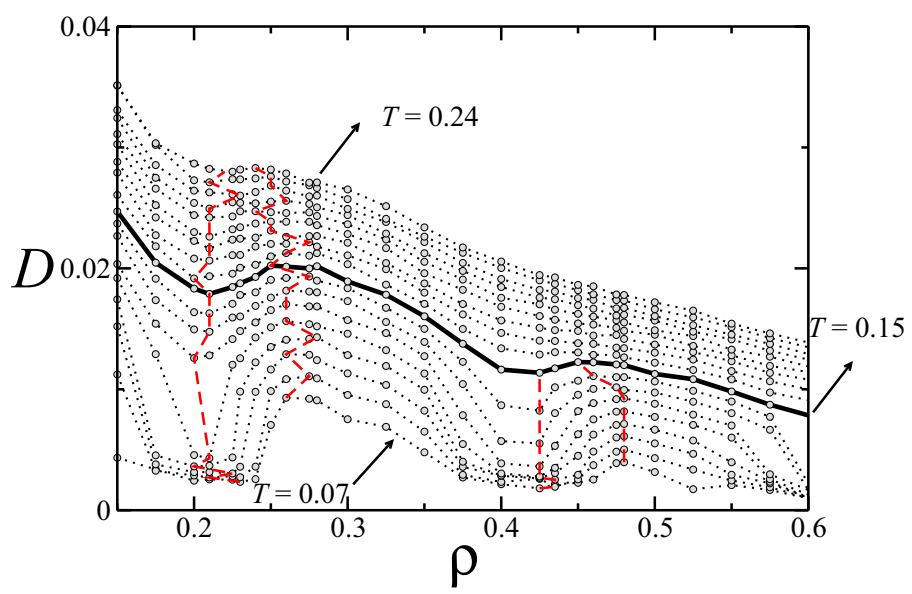

(b)

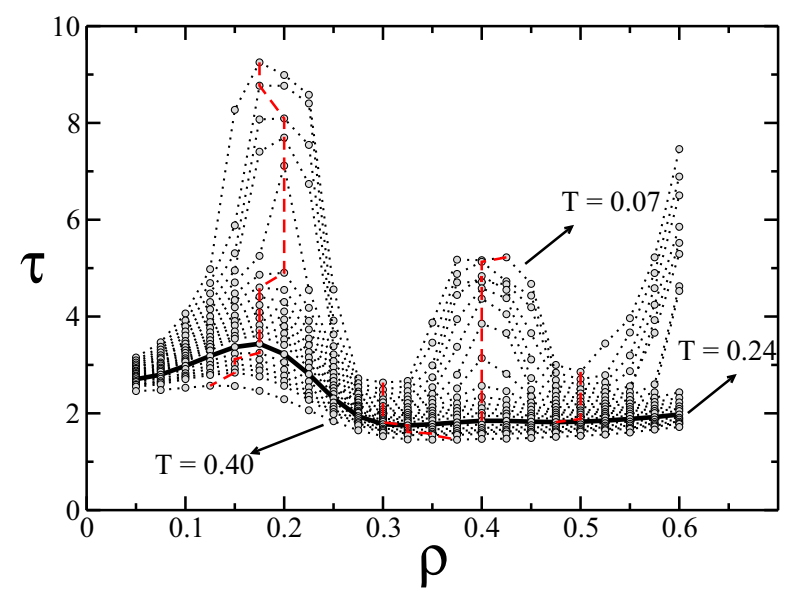

FIG. 2. (a) Diffusion constant $D$ and (b) translational order parameter $\tau$ as function of the system density. In both figures the maxima and minima that characterize the anomalies are represented by a dashed red line. For the diffusion anomaly, the anomalous region at lower densities ranges from the isotherm $T=0.07$ to $T=0.24$, while the second anomalous region goes from $T=0.07$ to $T=0.15$. In the case of the structural anomaly, the first anomalous regions goes from the isotherm $T=0.07$ to $T=0.40$, and the anomalous region at higher densities is located between the temperatures $T=0.07$ and $T=0.24$. The errors bars in $D$ and $\tau$ are smaller than the data point.

\section{RESULTS AND DISCUSSION}

For most fluids, the diffusion constant, $D$, decreases with the density, $\rho$. The reason for this behavior is that the particles become more structured as the density increases. Then the translational order parameter $\tau$, defined by Eq. (4), grows with $\rho$ as follows. At low densities, $g(r) \approx 1$ and then $\tau \approx 0$. As the density increases, $g(r) \neq 1$ for many values of $r$ and then $\tau$ grows. Anomalous fluids show the opposite behavior. For these materials in a certain range of temperature and pressures-the anomalous region-the diffusion coefficient increases with density and $\tau$ decreases with $\rho$. Figure 2(a) shows the dependence of the diffusion coefficient, $D$, with the density, $\rho$. As the density is increased from the gas phase, the diffusion coefficient decreases, reaches the first minimum in the density and then increases, reaching a the first maximum which characterizes the first anomalous region from the isotherm $T=0.07$ to the isotherm $T=0.24$. Then, as the density is increased even further, for the isotherms between $T=0.07$ and $T=0.15$, a second minimum and a second maximum are observed.

The translational order parameter versus density shown in Fig. 2(b) also indicates the existence of two anomalous regions in the pressure versus temperature phase diagram. The first is located between the isotherms $T=0.07$ and $T=0.40$ for low values of density, while the second occurs for higher densities and for the isotherm from $T=0.07$ to $T=0.24$.

Figure 3 shows the pressure versus temperature phase diagram, with the maximum and minimum of diffusion and translational order parameter shown as dotted-dashed and dashed lines respectively. The isochores are the gray lines. The temperature of maximum density is also shown in green.

For the 3D system the TMD region in the pressure versus temperature phase diagram is located inside the diffusion maxima and minima regions, which are inside the maxima and minima of the translational order parameter $[20,65]$ region.
This sequence is the same as that observed in water: the so-called waterlike hierarchy. Here, unlike the 3D molecular system, in the 2D Brownian system the hierarchy in the anomalies is distinct from the water case. This change in the hierarchy was already observed in others works, and it is

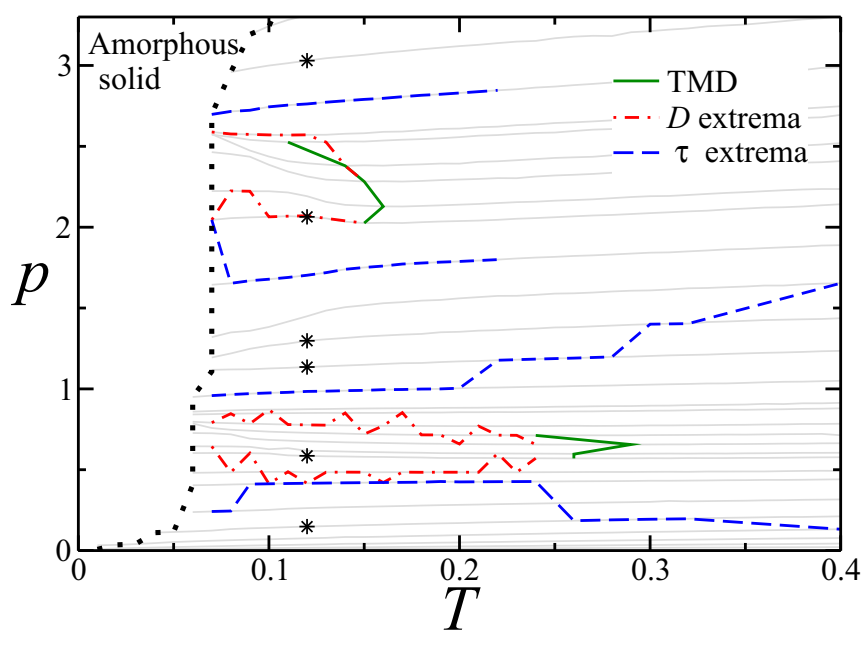

FIG. 3. $p \times T$ phase diagram of the colloidal system. The gray lines are the isochores. The dashed blue line delimits the structural anomaly regions, with the maximum and minimum values of $\tau$. The dotted-dashed red line delimits the diffusion anomalous regions, with the minimum and maximum values of $D$. The green line defines the density anomaly region and corresponds to the temperature of maximum density (TMD) line. The black stars are located over the isotherm $T=0.12$ and correspond to the densities $\rho=0.15$, $\rho=0.225, \rho=0.325, \rho=0.35, \rho=0.425$, and $\rho=0.525$. The dotted black line delimits the fluid and amorphous solid regions. The errors obtained for the mean value of $p$ and $T$ were smaller than $10^{-4}$ for all cases, and the errors bars were omitted for simplicity. 
(a)

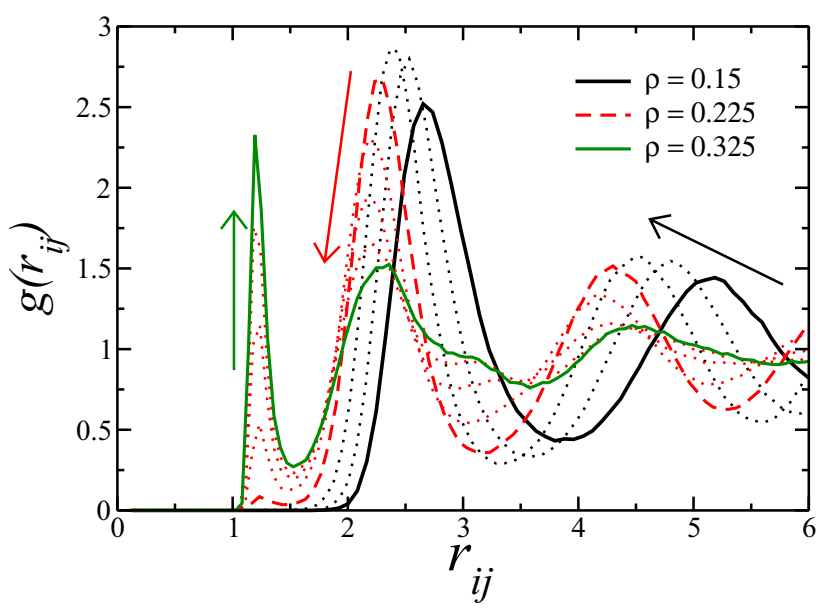

(c) (b)

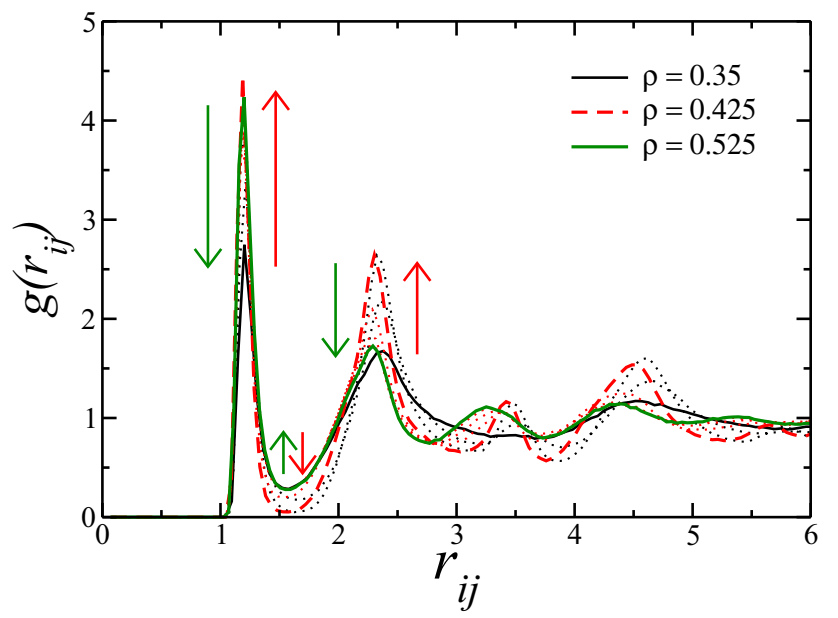

(d)

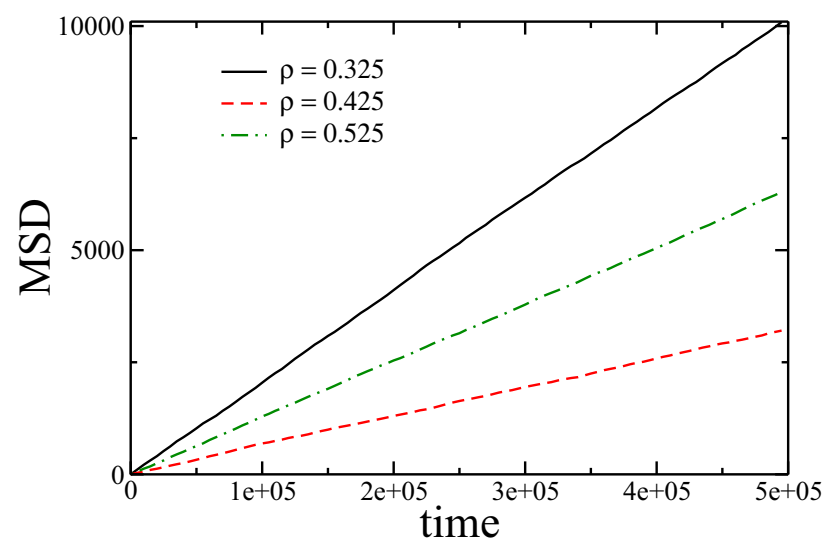

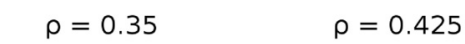

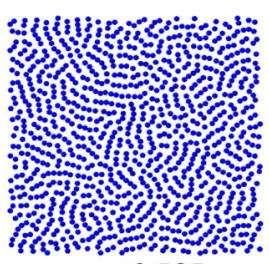

$\rho=0.525$

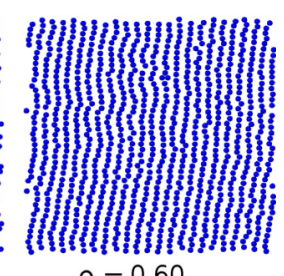

$\rho=0.60$

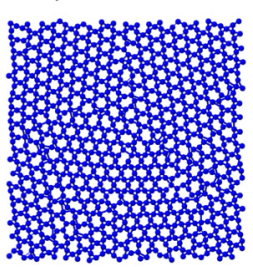

FIG. 4. Analysis of the isotherm $T=0.12$ for the colloidal system. (a) Radial distribution function (RDF) $g\left(r_{i j}\right)$ for densities inside the first anomalous region indicates that these anomalies originate by the competition between the two length scales. Curves for densities $0.15 \leqslant \rho<0.225$ are represented by black lines, for $0.225 \leqslant \rho<0.325$ by red lines, and for $\rho=0.325$ by the green line. The arrows shows how the peaks in $g\left(r_{i j}\right)$ move. The black arrow shows the grow of the second peak for densities below $\rho=0.225$, the red arrow the decrease in the second peak, and the green arrow the increase in the first peak for densities between $\rho=0.225$ and $\rho=0.325$. (b) Radial distribution function (RDF) $g\left(r_{i j}\right)$ for densities inside the second anomalous region indicates that there is not a competition between the scales. Curves for densities $0.35 \leqslant \rho<0.425$ are represented by black lines, for $0.425 \leqslant \rho<0.525$ by red lines, and for $\rho=0.525$ by the green line. Both peaks increase from $\rho=0.35$ to $\rho=0.425$, while the valley between them decreases. This is indicated by the red arrows. The green arrows show that from $\rho=0.425$ to $\rho=0.525$ the peaks decrease and the valley increases. Therefore, the system becomes more structured and then more disordered, which explains the second structural anomaly. Related to this transition from disordered to ordered to disordered structure, the slope of the MSD curve decreases and then increases, as shown in (c). The snapshots in (d) show the disks' conformation, including a kagome lattice at $\rho=0.60$.

attributed to the changes in the competition between the scales $[21,64,73]$, to the formation of an ordering structure [74], or to the dimensional change from $3 \mathrm{D}$ to $2 \mathrm{D}$ [75].

In our system, the change in the hierarchy is due to the presence of solidlike (or pinning-like) structures and to the change in the dimensionality, as shown next. In addition to the hierarchy, another question is also relevant: Why there are two anomalous regions in this system?

In order to understand the mechanism which generates the two anomalous regions, the behavior of the system at the isotherm $T=0.12$, shown as stars in the phase diagram of Fig. 3, is analyzed. In the case of the $3 \mathrm{D}$ system, the mechanism which explains the existence of the waterlike anomalies is the competition between the two length scales [25]. This is observed in the radial distribution function of molecular systems as follows.

In the anomalous region the first peak of the RDF increases with the density while the second peak decreases [30]. This behavior is also observed in Fig. 4(a), which corresponds to the low density and low pressure region of Fig. 3. In this region, as $\rho$ increases, particles move from the second length scale at $\approx 2.0$ to the first length scale at $\approx 1.2$. Therefore, the system has competition between the scales and, as a consequence, waterlike anomalies.

Figure 4(b) shows the RDF for densities bellow, inside, and above the second anomalous region. As the density is increased, the peaks of $g\left(r_{i j}\right)$ related to the first and second length scales increase, reach a maximum, and then decrease. 
Therefore, no competition between the two length scales is observed. However, the system shows density, diffusion, and structural anomalies. In order to reveal the origin of these anomalies, instead of looking to the two peaks it is necessary to examine the valley between them. As the density increases from $\rho=0.35$ to $\rho=0.425$ this valley goes down, becoming zero. These zeroes for the RDF suggests that as the density $\rho=0.425$ is approached from lower densities, the system is becoming solid, or well structured. The MSD, illustrated in Fig. 4(c), supports this result. The slope of the MSD decreases from $\rho=0.35$ to $\rho=0.425$, which indicates a decrease in the diffusion. This is reinforced by the snapshots shown in Fig. 4(d) as a stripe pattern. However, increasing the density even further to $\rho=0.525$, the disks becomes disordered. Despite the absence of competition, this behavior can also be understood based on the two-length-scales characteristics.

When the system is in the stripe structure, the interparticle distance between disks in the same stripe is the first length scale, and the stripes are separated by the second length scalethis is why both peaks increase from $\rho=0.35$ to $\rho=0.425$. At $\rho=0.425$ the particles have the minimum in the diffusion. Increasing the density, there is no more space for the stripes to remain at the distance $\approx 2.0$, and they break into the disordered fluid. Essentially, the enthalpic contribution to the free energy (second length scale) is overcome by the entropic contribution (the first length scale) [76]. Then the system goes from a disordered fluid to an ordered fluid (similar to a liquid crystal) with lower diffusion, and then gets disordered again, diffusing faster. In this reentrant melting region we observe that $D$ increases with the density while $\tau$ decreases, leading to the second anomalous regions. As the density increases even more, the system goes to a solid phase with a kagome lattice, as the snapshot in Fig. 4(d) shows.

Previous studies have shown that the existence of multiples competitive scales leads to multiples anomalous regions. In the work by Barbosa and co-workers [26,77], they used a soft-core potential with three characteristic length scales and found two TMD lines and transitions between three fluids phases. Also, we have observed two structural anomalous regions in quasi$2 \mathrm{D}$ systems, were the new anomalous regions can be related to the melting of the central layer between two walls [69]. This is similar to what we observed for the 2D system, were a reentrant melting region leads to the appearance of anomalies.

\section{CONCLUSION}

Langevin dynamics simulations of 2D core-softened disks were performed to analyze the system fluid phase for structural, thermodynamic, and dynamic anomalous behavior.

The core-corona colloidal system shows the presence of two anomalous regions in the pressure versus temperature phase diagram. Also, a change in the waterlike hierarchy of anomalies was observed, which can be associated with the change in the dimensionality.

The two distinct regions with anomalous behavior observed for the colloidal system arise due to two distinct mechanisms. The first region, at low densities, is associated with the competition between the two length scales in the interaction potential. This is the same mechanism observed in previous works and in the molecular system. We have shown that the second anomalous region is not related to the competition observed in the RDF, but to a reentrant fluid phase. This leads the fluid to suffer a transition from a disordered structure to an ordered structure and then back to a disordered structure, resulting in an increase in the diffusion as the density increases and a decrease of $\tau$ as $\rho$ increases - the anomalous behavior.

Nevertheless, this was not the first time that weobserved two anomalous regions for core-softened fluids. In a previous work, core-softened potentials with three scales led to two regions of density anomaly [26]. As well, we have shown that fluids modeled by potential equation (1) confined between two flat walls have a second structural anomaly. This new anomaly was not related to the competition between the potential scales, but to changes in the number of fluid layers between the walls [69]. The change in the number of layers is a additional competition induced by the confinement. In this work, the competition was induced by the resulting fluid reentrant phase. Therefore, our main finding is that other mechanisms, despite the competition between the scales, can generate competitions in the system that lead to waterlike anomalies.

\section{ACKNOWLEDGMENTS}

We thank the Brazilian agency CNPq (Grant No. 441712/2014-2) for the financial support. J.R.B. would like to thanks Professor Alexandre Diehl from Universidade Federal de Pelotas for the computational time in the TSSC cluster.
[1] M. Chaplin, Seventy-three anomalies of water, http://www.lsbu.ac.uk/water/anmlies.html (2015).

[2] P. A. Netz, F. W. Starr, M. C. Barbosa, and H. E. Stanley, Physica A (Amsterdam) 314, 470 (2002).

[3] T. Morishita, Phys. Rev. E 72, 021201 (2005).

[4] S. Sastry and C. A. Angell, Nat. Mater. 2, 739 (2003).

[5] G. S. Kellu, J. Chem. Eng. Data 20, 97 (1975).

[6] R. Sharma, S. N. Chakraborty, and C. Chakravarty, J. Chem. Phys. 125, 204501 (2006).

[7] H. Thurn and J. Ruska, J. Non-Cryst. Solids 22, 331 (1976).

[8] Handbook of Chemistry and Physics, 65th ed. (CRC, Boca Raton, FL, 1984).

[9] S. J. Kennedy and J. C. Wheeler, J. Chem. Phys. 78, 1523 (1983).
[10] T. Tsuchiya, J. Phys. Soc. Jpn. 60, 227 (1991).

[11] P. T. Cummings and G. Stell, Mol. Phys. 43, 1267 (1981).

[12] M. Togaya, Phys. Rev. Lett. 79, 2474 (1997).

[13] C. A. Angell, R. D. Bressel, M. Hemmatti, E. J. Sare, and J. C. Tucker, Phys. Chem. Chem. Phys. 2, 1559 (2000).

[14] E. A. Jagla, Phys. Rev. E 58, 1478 (1998).

[15] E. A. Jagla, J. Chem. Phys. 110, 451 (1999).

[16] E. A. Jagla, J. Chem. Phys. 111, 8980 (1999).

[17] G. Malescio, G. Franzese, A. Skibinsky, S. V. Buldyrev, and H. E. Stanley, Phys. Rev. E 71, 061504 (2005).

[18] A. Scala, F. W. Starr, E. La Nave, F. Sciortino, and H. E. Stanley, Nature (London) 406, 166 (2000). 
[19] L. Xu, P. Kumar, S. V. Buldyrev, S. H. Chen, P. Poole, F. Sciortino, and H. E. Stanley, Proc. Natl. Acad. Sci. USA 102, 16558 (2005).

[20] A. B. de Oliveira, P. A. Netz, T. Colla, and M. C. Barbosa, J. Chem. Phys. 124, 084505 (2006).

[21] Y. D. Fomin, E. N. Tsiok, and V. N. Ryzhov, J. Chem. Phys. 135, 234502 (2011).

[22] Z. Yan, S. V. Buldyrev, N. Giovambattista, and H. E. Stanley, Phys. Rev. Lett. 95, 130604 (2005).

[23] D. Y. Fomin, N. V. Gribova, V. N. Ryzhov, S. M. Stishov, and D. Frenkel, J. Chem. Phys. 129, 064512 (2008).

[24] E. Lascaris, G. Malescio, S. V. Buldyrev, and H. E. Stanley, Phys. Rev. E 81, 031201 (2010).

[25] A. B. de Oliveira, P. A. Netz, and M. C. Barbosa, Physica A (Amsterdam) 386, 744 (2007).

[26] M. A. Barbosa, E. Salcedo, and M. C. Barbosa, Phys. Rev. E 87, 032303 (2013).

[27] F. Saija, S. Prestipino, and G. Malescio, Phys. Rev. E 80, 031502 (2009).

[28] G. Malescio and F. Saija, J. Phys. Chem. B 115, 14091 (2011).

[29] S. Prestipino, F. Saija, and G. Malescio, J. Chem. Phys. 133, 144504 (2010).

[30] N. M. Barraz Jr., E. Salcedo, and M. C. Barbosa, J. Chem. Phys. 131, 094504 (2009).

[31] N. B. Wilding and J. E. Magee, Phys. Rev. E 66, 031509 (2002).

[32] G. Franzese, M. I. Marqués, and H. E. Stanley, Phys. Rev. E 67, 011103 (2003).

[33] S. Buldyrev, G. Franzese, N. Giovambattista, G. Malescio, M. Sadr-Lahijany, A. Scala, A. Skibinsky, and H. Stanley, Physica A (Amsterdam) 304, 23 (2002).

[34] A. M. Almudallal, S. V. Buldyrev, and I. Saika-Voivod, J. Chem. Phys. 137, 034507 (2012).

[35] T. Urbic, Phys. Rev. E 88, 062303 (2013).

[36] P. H. Poole, F. Sciortino, U. Essmann, and H. E. Stanley, Nature (London) 360, 324 (1992).

[37] T. Morishita, Phys. Rev. Lett. 87, 105701 (2001).

[38] I. Saika-Voivod, F. Sciortino, and P. H. Poole, Phys. Rev. E 63, 011202 (2000).

[39] E. Lascaris, M. Hemmati, S. V. Buldyrev, H. E. Stanley, and C. A. Angell, J. Chem. Phys. 140, 224502 (2014).

[40] V. V. Vasisht, S. Saw, and S. Sastry, Nat. Phys. 7, 549 (2011).

[41] J. N. Glosli and F. H. Ree, Phys. Rev. Lett. 82, 4659 (1999).

[42] S. Scandolo, Proc. Natl. Acad. Sci. USA 100, 3051 (2003).

[43] F. Smallenburg, L. Filion, and F. Sciortino, Nat. Phys. 10, 653 (2014).

[44] M. Quesada-Perez, A. Moncho-Jorda, F. Martinez-Lopez, and R. Hidalgo-Álvarez, J. Chem. Phys. 115, 10897 (2001).

[45] C. Contreras-Aburto and J. M. amd R. C. Priego, J. Chem. Phys. 132, 174111 (2010).

[46] G. Malescio and G. Pellicane, Nat. Mater. 2, 97 (2003).

[47] P. Camp, Phys. Rev. E 68, 061506 (2003).

[48] J. Fornleitner and G. Kahl, J. Phys.: Condens. Matter 22, 104118 (2010).
[49] M. Singh, H. Liu, S. K. Kumar, A. Ganguly, and C. Chakravarty, J. Chem. Phys. 132, 074503 (2010).

[50] C. I. Mendoza and E. Batta, Europhys. Lett. 85, 56004 (2009).

[51] H. Pattabhiraman, A. P. Gantapara, and M. Dijkstra, J. Chem. Phys. 143, 164905 (2015).

[52] H. Pattabhiraman and M. Dijkstra, Soft Matter 13, 4418 (2017).

[53] H. Pattabhiraman and M. Dijkstra, J. Chem. Phys. 146, 114901 (2017).

[54] H. G. Schoberth, H. Emmerich, M. Holzinger, M. Dulle, S. Forster, and T. Gruhn, Soft Matter 12, 7644 (2016).

[55] H. J. Zhao, V. R. Misko, and F. M. Peeters, New J. Phys. 14, 063032 (2012).

[56] H. J. Zhao, V. R. Misko, and F. M. Peeters, Phys. Rev. E 88, 022914 (2013).

[57] A. Ciach and J. Pekalski, Soft Matter 13, 2603 (2017).

[58] J. R. Bordin, Physica A (Amsterdam) 495, 215 (2018).

[59] S. Prestipino, F. Saija, and P. V. Giaquinta, J. Chem. Phys. 137, 104503 (2012).

[60] D. E. Dudalov, E. N. Tsiok, Y. D. Fomin, and V. N. Ryzhov, J. Chem. Phys. 141, 18C522 (2014).

[61] E. Tsiok, Y. Fomin, and V. Ryzhov, Physica A (Amsterdam) 490, 819 (2018).

[62] J. R. Bordin and L. B. Krott, Phys. Chem. Chem. Phys. 16, 28740 (2016).

[63] L. B. Krott, C. Gavazzoni, and J. R. Bordin, J. Chem. Phys. 145, 244906 (2016).

[64] J. R. Bordin, Physica A (Amsterdam) 459, 1 (2016).

[65] A. B. de Oliveira, P. A. Netz, T. Colla, and M. C. Barbosa, J. Chem. Phys. 125, 124503 (2006).

[66] L. B. Krott and M. C. Barbosa, J. Chem. Phys. 138, 084505 (2013).

[67] L. Krott and J. R. Bordin, J. Chem. Phys. 139, 154502 (2013).

[68] L. B. Krott, J. R. Bordin, N. Barraz, Jr., and M. C. Barbosa, J. Chem. Phys. 1, 134502 (2015).

[69] L. B. Krott, J. R. Bordin, and M. C. Barbosa, J. Phys. Chem. B 119, 291 (2015).

[70] M. P. Allen and D. J. Tildesley, Computer Simulation of Liquids (Oxford University Press, Oxford, 1987).

[71] A. B. de Oliveira, E. Salcedo, C. Chakravarty, and M. C. Barbosa, J. Chem. Phys. 142, 234509 (2010).

[72] J. R. Errington and P. D. Debenedetti, Nature (London) 409, 318 (2001).

[73] J. R. Bordin and L. B. Krott, J. Phys. Chem. B 121, 4308 (2017).

[74] M. Girardi, M. Szortyka, and M. C. Barbosa, Physica A (Amsterdam) 386, 692 (2007).

[75] D. E. Dudalov, Y. D. Fomin, E. N. Tsiok, and V. N. Ryzhov, Soft Matter 10, 4966 (2014).

[76] J. R. Bordin, A. B. de Oliveira, A. Diehl, and M. C. Barbosa, J. Chem. Phys. 137, 084504 (2012).

[77] E. O. Rizzatti, M. A. A. Barbosa, and M. C. Barbosa, Frontiers Phys. 13, 136102 (2018). 